\title{
VIDEOJUEGOS Y OCIO: ALGUNAS REFLEXIONES SOBRE SU INCIDENCIA EN EL DESARROLLO FÍSICO-SOCIAL DE LOS JÓVENES
}

\section{VIDEO GAMES AND LEISURE: REFLECTIONS ON ITS IMPACT IN THE PHYSICAL AND SOCIAL DEVELOPMENT OF YOUTH}

Mónica Patricia Melo Herrera ${ }^{1}$

Rubinsten Hernández Barbosa²

\section{Resumen}

Los diferentes usos que los videojuegos tienen en la actualidad, especialmente entre la población infantil y juvenil, y la manera como estos se relacionan, no solo en los espacios educativos formales y no formales, sino también con el ocio, han generado una serie de cuestionamientos entre los autores, los cuales presentan el siguiente artículo con el objetivo de compartir algunas reflexiones que se hacen sobre su uso, basados en un análisis desde diferentes perspectivas. De esta manera, se describe la incidencia de los videojuegos en el desarrollo social, psicológico y físico; finalmente, se considera algunos aspectos sobre su manejo responsable. Además de lo anterior, el presente estudio también es una manera de invitar a las diferentes comunidades a reflexionar sobre el papel que tienen sobre el empleo de este recurso tecnológico.

Palabras claves: videojuegos; ocio; tiempo libre; aprendizaje

\section{Abstract}

The different uses that video games have today, especially among children and young people, and the way they relate, not only in formal and non-formal educational spaces, but also in leisure generated a series of questions among the authors, which have written the following article in order to share some thoughts around their use, based on an analysis from different perspectives. Thus, the impact of video games on the social, psychological and physical development is described; finally, it considers some aspects of their responsible management. In addition, it also an invitation for different communities to reflect on the role they have on the use of this technological resource.

Keywords: games; entertainment; leisure; learning

Fecha de recepción: 20 de octubre de 2015

Fecha de aprobación: 8 de marzo de 2016

Para citar este artículo:

Melo, M.P. y Hernández, R. (2016). Video juegos y ocio: algunas reflexiones sobre su incidencia en el desarrollo físico-social de los jóvenes. Lúdica Pedagógica, (23), 85-91.

1 Magíster en Didáctica de las Ciencias, especialista en Gestión de Proyectos, administradora deportiva. Docente de la Secretaría de Educación de Bogotá. Correo electrónico: ud2000admon@yahoo.com

2 Docente investigador. Universidad Autónoma de Colombia. Correo electrónico: rhbjd@hotmail.com 


\section{INTRODUCCIÓN}

El desarrollo de las tecnologías de la información y la comunicación (TIC) ha permeado la gran mayoría de los aspectos de la vida del ser humano: sus relaciones sociales, el trabajo, las conversaciones con la familia y el ocio, entre otros, los cuales de una u otra manera se están generando por medios virtuales. Las grandes empresas han aprovechado estas necesidades de asociación y comunicación para llevar al mercado una gran variedad de recursos tecnológicos entre los que se encuentra los videojuegos, han aprovechado, asimismo, que el ocio electrónico surge de la necesidad de emplear el tiempo que se tiene en los diferentes espacios y momentos de las personas, por lo que se ha convertido en uno de los negocios más rentables de la actualidad.

En la actualidad, el uso de estos videojuegos, especialmente por la población juvenil, viene aumentando de manera vertiginosa como una forma de ocio recreativo. Esa necesidad de jugar, que es natural en esta etapa de la vida, hace que los jóvenes pasen horas y horas frente a un ordenador o un móvil, sin pensar en el uso apropiado de estos recursos. Debido a este uso inadecuado, se han presentado problemas de orden físico como el aumento de peso y obesidad en ciertos casos; también, algunos estudios reportan dificultades sociales que pueden generar los videojuegos, como el aislamiento, lo cual conduce a sus usuarios, en ocasiones, a desarrollar conductas agresivas con familiares y amigos. Estas consecuencias negativas son razones suficientes para pensar en la necesidad de fomentar un uso responsable de los videojuegos, ya que en esta actividad están involucrados no solo el niño o joven que juega, sino también los individuos con los que se relaciona en su cotidianidad: padres de familia, amigos y profesores.

Según lo dicho, y considerando la importancia del tema, se comparten algunas reflexiones que los autores han hecho teniendo como marco el contexto educativo y recreativo. A partir de estos análisis, se realizan una serie de conclusiones y sugerencias en torno al uso adecuado de los juegos de video, no solo para la persona que juega ya que son muchos los actores sociales los llamados a reflexionar sobre las prácticas que los jóvenes hacen en su tiempo de ocio.

\section{APRENDIENDO CON LOS VIDEOJUEGOS EN LA ESCUELA}

Desde los procesos de aprendizaje, los juegos se han convertido en herramientas que facilitan los procesos pedagógicos, tanto de docentes como de estudiantes; con su práctica, el jugador puede generar una variedad de habilidades en lo que tiene que ver con relaciones entre sus conocimientos previos, resolución de conflictos, toma de decisiones, diseño de estrategias, concentración, desarrollo motor. Estos son algunos de los fines que la educación pretende fomentar en las personas, con el valor agregado que el jugador se divierte, lo disfruta, no tiene en cuenta el tiempo empleado, y se convierte así "en un aliado de las actividades en el aula [...] por su carácter motivante y divertido, pues al mismo tiempo que se aprende, se estimulan las capacidades del pensamiento, la creatividad y el espíritu investigativo del niño" (Melo y Hernández, 2014, p. 59).

En este sentido, el juego se ha visto inmerso en el fenómeno de las Tic el cual ha logrado en los últimos años el nacimiento de una gran variedad de recursos adaptables a escenarios pedagógicos como los blogs, páginas web, ambientes virtuales de aprendizaje y el boom de los videojuegos, que han brindado tanto al educador como al educando la posibilidad de experimentar la educación virtual, en todas las áreas del conocimiento y con contenidos variados, tanto en forma como en fondo, y que han permitido en algunos casos que los aprendizajes sean más agradables debido a su interactividad y diseño.

Numerosas investigaciones han apuntado a que los videojuegos pueden favorecer significativamente los procesos de aprendizaje de las personas que juegan. Estos son una realidad innegable y latente, sus efectos deben ser estudiados desde una mirada crítica y reflexiva en el entorno educativo como práctica social que influencia la manera de pensar y actuar del estudiante; desde el punto de vista vigotskyano, se podría hablar de una herramienta que posibilita la interacción social y la construcción de conocimiento con otros miembros de su comunidad (Vigotsky, 2000).

Estudios han encontrado en el juego un carácter potencializador del aprendizaje y la construcción de conocimiento. Gros (2008), al dirigir el grupo F9 de la Universidad Autónoma de Barcelona argumenta que se pueden generar múltiples posibilidades en la educación al emplear consolas de juego y artefactos electrónicos. Se basa, en parte, en los logros que ha obtenido con el 
juego Age of empire que se encuentra comercialmente y con el cual ha enseñado aspectos relacionados con imperios como el griego, inca y romano, al mismo tiempo que enseñó elementos de economía, geografía e historia. Sin embargo, los estudiosos consideran que los juegos educativos carecen de esa capacidad motivadora e interesante que tienen los juegos que se encuentran en el mercado.

En este mismo sentido, Garrido (2013) evidenció los aspectos favorecedores de los videojuegos de estrategia en la enseñanza bajo la implementación de estos en el aula. Sus hallazgos fueron satisfactorios al encontrar que los estudiantes se sienten más involucrados al participar de un sistema organizado, lo que le permitía tomar decisiones. Por otro lado, resultó llamativo y desafiante debido a las situaciones cambiantes que debían superar identificando el qué, cómo, cuándo y dónde debían actuar en el escenario virtual. Finalmente, se observó el alto nivel de satisfacción de los participantes cuando lograban alcanzar los objetivos del juego.

\section{VIDEO JUEGOS Y TIEMPO DE OCIO EN LA POBLACIÓN JUVENIL}

Aunque en los procesos educativos los videojuegos se han convertido en una herramenta aliada para favorecer los aprendizajes en el aula, estos son usados con mayor frecuencia e intensidad horaria en los tiempos de ocio, entendido este como "el comportamiento que se caracteriza por consumir y emplear una parte del tiempo de que se dispone tanto cada persona como el sistema social" (Munné y Cordina, 1996, p. 430), el cual se centra en realizar actividades para el disfrute personal y colectivo.

En este sentido, Marqués (2000) señala que los videojuegos, además de ser motivadores, favorecen la destreza manual y organizativa debido a que los practicantes son autónomos en su asignación y al manejo de los controles; también favorece la toma de decisiones y la cooperación. Este sugiere que de acuerdo a la clase de juego que se emplee se puede potenciar capacidades y habilidades concernientes al aprendizaje, por ejemplo, los ahora Nintendo Wii favorece la coordinación psicomotriz, orientación espacial y agilidad mental entre otros. Los simuladores favorecen el espíritu investigativo, la estrategia y la resolución de problemas.
El uso de los videojuegos en los tiempos de ocio de los jóvenes ha originado que se tenga una mirada distinta de su papel en el mundo; el alto volumen de información visual que encuentran en este recurso presupone que debe haber una alfabetización digital, ya que se pueden convertir en una fuente peligrosa de información, debido a que la preferencia de los jóvenes se centran en juegos con contenidos altamente violentos, los cuales inciden en su desarrollo. Investigaciones, como las de Estallo (1995), citado en Llorca (2006), muestran como dato importante que

El tiempo que dedican los menores a los videojuegos y a la televisión es mayor que el que dedican a la escuela, el trayecto al colegio y a la realización de las tareas escolares, tal y como se viene confirmando a lo largo de casi todos los estudios que se realizan sobre los hábitos diarios de niños y adolescentes. (p. 81).

Estos datos muestran la alta influencia que ejercen los videojuegos en la población juvenil, los cuales desencadenan un desinteres por actividades propias de su edad como la escuela, la familia, los amigos, entre otros. Los jovenes se han adaptado con facilidad a esta realidad hecha para sus intereses y necesidades, cargadas de aventura, misterio, que les brinda la posibilidad de modificar en cualquier momento la misma; con lo que los videojuegos se han posicionado como una de las primeras opciones para pasar su tiempo de ocio; con el agravante de que no se tiene un uso moderado, un control y una selección de los mismos.

\section{EFECTOS COLATERALES DEL USO DE LOS VIDEOJUEGOS}

Si bien es cierto que los videojuegos han favorecido de cierta manera el desarrollo de los jóvenes, no todo lo que se ve es positivo. A pesar de que existen experiencias enriquecedoras, tanto en el contexto educativo como en el social, también se debe hablar de la otra cara de la moneda, los posibles riesgos que estos generan en la población que los juega, desde diferentes puntos de vista como el comportamental, social, cultural y de salud, entre otros.

De la misma manera que numerosos estudios han encontrado aspectos favorables en la práctica de los videojuegos, también se han hallado aspectos desfavo- 
rables debido a su uso inadecuado y desmedido, tanto en la vida cotidiana como en el aula, lo que ha motivado una profunda reflexión en ambientes escolares y sociales.

El aumento vertiginoso del consumo de los videojuegos diseñados para niños y adolescentes, incluso para adultos, sugiere que se han convertido en los juguetes favoritos de esta generación, lo que ha conllevado a que su uso sea un reto tanto para padres como para educadores debido a su reciente y creciente incursión en las aulas de clase y a su aumento en el consumo de su tiempo de ocio y que se hace presente en todos los contextos socioculturales, lo que sugiere un cambio de actitud y usabilidad de este recurso tecnológico.

Frente a este fenómeno se han realizado estudios que evidencian su incidencia en la conducta de la persona que juega. La organización Protégeles, citada en Balerdi (2011), ha realizado minuciosas investigaciones de los efectos que produce el uso desmedido de los videojuegos; menciona en varios informes que en el libro Videojuegos, guía de padres (2005) se revelan las cifras, no muy alentadoras, de un estudio hecho en España:

- Casi el $50 \%$ de los niños y el $25 \%$ de las niñas reconoce que si sus padres conocieran el contenido de algunos de sus videojuegos no les dejarían jugar con ellos.

- Más del 50 \% de los niños y $15 \%$ de las niñas reconoce que juega con videojuegos clasificados para mayores de 18 años.

- El $73 \%$ de los niños y el $40 \%$ de las niñas juegan con videojuegos violentos.

- Un $28 \%$ de los niños y un $3 \%$ de las niñas juegan con videojuegos en los que los personajes consumen drogas.

- Un $15 \%$ de los niños y un $7 \%$ de las niñas consideran que los videojuegos pueden hacerles más violentos. (p. 34).

Estas cifras denotan que la población infantil y juvenil está siendo la más afectada con el contenido de los videojuegos. Otro aspecto que se destaca es que hay un desentendimiento del grupo familiar, los cuales no prestan atención ni orientan a sus hijos a un buen manejo de estos recursos, también por el desconocimiento de ellos hacia los contenidos de los juegos de video; clara muestra de que el desarrollo tecnológico viene permeando la formación integral de las nuevas generaciones y las lleva a adquirir comportamientos que atentan en contra de su integridad emocional y física y de su ambiente comunitario.

Por otro lado, esta asociación evidenció, en uno de sus estudios, que "el 57 \% de los menores ha reconocido que juega con videojuegos en los que se daña, tortura o mata a las personas y que en un $20 \%$ de los casos se corresponden con niños, ancianos o embarazadas" (Balerdi, 2011, p. 34). En este mismo sentido la CECU (Confederación Estatal de Consumidores de España) ha realizado varias denuncias de distintos videojuegos debido a sus escenas violentas, trato sexista y discriminatorio, y consumo de drogas.

Siguiendo la constante preocupación de las organizaciones mundiales, se destaca el llamado que hizo la APA (American Psychological Association) en el año 2005 a la reducción de los tratos agresivos en los videojuegos, ya que se identificó que este contenido estaba influenciando la agresión hacia las mujeres. La AAP (American Academy of Pediatrics) reconoce que la exposición violenta de las Tic está afectando significativamente la salud de niños y adolescentes en términos tanto físicos como psicológicos.

Otro aspecto que viene afectando el uso inadecuado y excesivo de los videojuegos se orienta al aislamiento social. Actualmente, los jóvenes prefieren estar solos jugando en vez de pasar tiempo con amigos o familiares, se están volviendo introvertidos, se refugian en sus actividades virtuales, pierden la noción de su mundo, en algunos casos no les importa su presentación personal y su aseo debido a que al jugar con una consola en su casa nadie lo ve ni lo critica. De la Cruz (2002) ha realizado una recopilación de estudios minuciosos de los efectos de los videojuegos en todas las aristas del ser humano, y ha encontrado datos interesantes que se pueden considerar como una alerta, un llamado a realizar un cambio en su uso, a lo que el autor sugiere:

"La alarma se debe disparar cuando, más allá de la distracción, el juego cumple la función de "refugio". El microcosmos de los videojuegos parece haberse convertido en un cierto mundo aparte que "no sólo excluye a los de afuera, sino que también aísla a los de adentro. Para Maressa Hecht, de los servicios de Adicción a los Ordenadores de McLean Hospital, un centro afiliado a la universidad de Harvard (www.computeraddiction.com), los jugadores en 
su mayoría son personas solitarias, que en el mundo virtual se relacionan con sus únicos amigos". (De la Cruz, 2002, p. 15).

\section{INCIDENCIA EN EL DESARROLLO FÍSICO}

No solo el aspecto emocional y conductual de la persona que juega se ve afectada por el uso de los videojuegos; también se han visto que su condición física se deteriora a causa del sedentarismo que estos producen, ya que son actividades pasivas que requieren un mínimo de movimientos corporales. En el caso del uso excesivo de los videojuegos en la población escolar, se han descrito problemas oculares, auditivos, dolor de cabeza, inflamación en tendones, músculos, manos y muñeca y lesiones en el pulgar (Gil y Vida, 2007, p. 45). Estos problemas de salud, resultantes del uso desmedido de los videojuegos, inciden en el bajo rendimiento académico de la población escolar, ya que es mayor el tiempo que emplean para su diversión, y dejan de lado las labores académicas.

Estos efectos lesivos para el cuerpo humano suponen un deterioro prematuro de algunos órganos, como por ejemplo la vista, ya que esta se esfuerza por largas horas sin que el jugador tenga conciencia de ello a causa del poder adictivo de estos juegos. Por otro lado, al emplear con mayor frecuencia las manos y el estar sentado por largos periodos de tiempo, se han originado enfermedades tempranas del túnel del carpo, tendinitis, problemas de columna y articulaciones, puesto que se minimiza la exigencia del movimiento corporal, lo que acarrea estas consecuencias.

Debido al aumento de la inactividad física en niños y adolescentes, también han comenzado a generarse problemas de insomnio y alteraciones del sueño (Rojas, 2008); la hora de dormir la han cambiado por la de jugar en el computador o en su consola de videojuegos, al mismo tiempo que el tiempo de hacer tareas lo emplean para dormir, en la mayoría de los casos; además, prefieren comer en su habitación que en el comedor y les parece mejor las comidas rápidas.

En este mismo sentido, se ha comprobado que los problemas de obesidad en la población infantil y juvenil han venido en aumento a causa del uso desmedido de los videojuegos, adicional a la mala alimentación; es de suponerse, si en vez de salir al parque con los amigos o a la calle a jugar pelota prefieren estar al frente de un televisor con el videojuego mientras se pierde la noción del tiempo y si en lugar de los espacios destinados para la cena familiar prefieren una comida rápida y continuar jugando. Según los especialistas, "mantener hábitos sedentarios como ver televisión y pasar prolongadas horas frente a los videojuegos o navegando en Internet, impiden a los jóvenes mantener un peso normal" (Ayela, 2009 , p. 70), lo que conduce a serios problemas en las articulaciones, músculos y circulatorios, entre otros, y repercute en la salud emocional.

\section{EL USO RESPONSABLE DE LOS VIDEOJUEGOS}

Teniendo en cuenta los factores descritos anteriormente, es fundamental hablar del uso responsable de los videojuegos y del acompañamiento de los mismos, ya que este fenómeno no es solo tema del que juega, las personas responsables de los jóvenes, los educadores, la sociedad en general, también tienen un papel significativo en el correcto empleo de estas herramientas tecnológicas. Los jugadores son seres sociales, sociables, y como tal, afecta al que está a su lado, de ahí la necesidad de realizar una alfabetización digital para jóvenes, docentes y cuidadores, al mismo tiempo que los medios comerciales deben regular su producción, de forma que se llame a una toma de conciencia del correcto uso de los juegos de video, su adecuada selección, su mesurada disposición de tiempo, entre otros.

Se resaltan las diferentes campañas que se han hecho al respecto, como las del sistema de clasificación de videojuegos PEgi (PAN EuRopeAn GAME INFORMATION), con las cuales se establecen unos criterios para la venta de estos juegos, de acuerdo a las edades adecuadas para su tipología; los códigos que se han diseñado para este sistema "viene acompañado por recomendaciones como la necesidad de que los videojuegos se utilicen asesorados por una persona adulta, para disfrutar junto a ellos de su tiempo de ocio" (Llorca, 2006, p. 83).

Debido a que algunos estudios han demostrado que existe una fuerte incidencia de los videojuegos en la conducta, Amnistía Internacional (AI), entidad encargada de velar por la protección de los derechos humanos, ha reconocido en sus informes (2007) que la influencia de los videojuegos trascienden a la vulneración de algunos derechos de los niños y adolescentes, indica, además, 
que a pesar de muchos controles, los fabricantes y vendedores siguen omitiendo la regulación ya existente, lo que impide un uso adecuado de este recurso tecnológico.

Las alarmas se han encendido en muchos lugares del mundo, se han generado mecanismos de denuncia, control, inspección y regulación de la comercialización de los juegos de video; la Confederación Estatal de Consumidores y Usuarios Española (CECU) durante años ha realizado llamados de atención en cuanto al contenido poco apto de ciertos videojuegos debido a que tienen contenido violento, como el matar a población vulnerable indistintamente; de discriminación, como los que el objetivo es acabar con la población de color de piel diferente a la del usuario, consumo de drogas, entre otros (Asociación Española de Distribuidores y Editores de Software de Entretenimiento, ADESE, 2010).

A pesar de esta clase de mecanismos preventivos, no han sido suficientes los esfuerzos para mitigar los problemas que se dan con el uso inadecuado de los videojuegos. El estilo de vida de la población juvenil ha cambiado drásticamente; su tiempo de ocio se orienta al uso desmedido de los videojuegos, situación que debe ser tratada desde todas las aristas de una sociedad de consumo; si el joven tiene acceso a un sinnúmero de juegos con contenidos que atentan contra su formación integral, no solo el cuidador debe velar por su protección, las empresas comercializadoras de estos productos deben encausar su nicho de mercado a fin de proteger a la población infantil y juvenil; los medios audiovisuales que invitan a su uso deben tener una regulación por parte de las entidades encargadas de este proceso, se debe hacer una alfabetización digital para padres y educadores, todos los actores involucrados deben velar por un buen uso de este recurso tecnológico.

\section{CONCLUSIONES}

Si bien es cierto que los juegos de video han ayudado en algunos aspectos de los resultados académicos y de formación de los educandos en diferentes campos, también hay que considerar los efectos que estos están generando por no usarlos de manera correcta y controlada, situación que viene creciendo sin un control real por parte de la mayoría de los sectores sociales.

Se resaltan los procesos de innovación pedagógica que se adelantan en las aulas de clase con la inclusión de estos recursos tecnológicos, donde se evidencia el tra- bajo de docente en cuanto al diseño y contenido de los juegos empleados para su quehacer en el aula, además de reconocer que el uso de las TIC es inevitable en todos los aspectos de la vida de las personas. Los buenos resultados que se han obtenido ponen de manifiesto el potencial pedagógico y de aprendizaje de los juegos de video en cuanto al desarrollo de habilidades, destrezas, valores, conocimientos; el jugador no solo mueve sus manos, también tiene una interacción psicológica y emocional, por lo cual el maestro está aprovechando su amplia gama de posibilidades.

En lo que respecta al estado físico y de salud de los jóvenes jugadores, se puede observar que, aunque en su gran mayoría los videojuegos promueven el sedentarismo, existen alternativas digitales que permiten el movimiento corporal, como los Nintendo Wii, con los cuales se están minimizando los riesgos de sufrir enfermedades a edades tempranas; es por ello que se debe incentivar en la población juvenil la reflexión en torno a los efectos del uso de estos juegos en sus tiempos de ocio.

\section{DISCUSIÓN}

Aunque es evidente que existen leyes que pretenden regular el uso de los videojuegos de acuerdo a ciertas edades, también es claro que no todas las personas respetan esta normatividad, especialmente los distribuidores y vendedores de estos recursos electrónicos. Si bien es cierto que se habla ahora de una sociedad digital, también es cierto que no se puede negar la naturaleza del ser humano como eso, como un ser que vive y convive con otros seres con los cuales se debe relacionar a diario y los cuales poseen unos derechos que no pueden ser violentados solo por el deseo de seguir convirtiendo la industria del entretenimiento como las primeras en la esfera económica.

En lo que respecta a los juegos de contenidos violentos, se debe no solo controlar y restringir su uso de acuerdo a las edades, hay que buscar todas las posibilidades pedagógicas, también se pueden emplear para el análisis de situaciones desafiantes en las que se puede ver envuelto un sujeto con el fin de aportar a su formación humana. Si existen juegos en los que se matan niños, mujeres embarazadas y ancianos, se puede recurrir a un proceso de sensibilización para mitigar los efectos de estos videos, promoviendo, al contrario, conductas de colaboración y ayuda. 
Por otro lado, las dificultades de salud física de los jugadores que se generan con algunos juegos de video, se pueden mitigar con nuevos juegos donde se tenga que emplear el movimiento corporal y participar activamente durante todo el tiempo de uso; como se mencionó, el Nintendo Wii es una buena alternativa, en este se participa de deportes de bajo impacto o extremos, como el esquí, donde el jugador debe ejecutar todos los movimientos corporales propios del deporte. Se puede, así, combatir el sedentarismo y los problemas de obesidad, sin embargo, es importante emplear mecanismos de sensibilización en la población juvenil.

Finalmente, en una sociedad de consumo y capitalista no se puede acarrear la carga únicamente a los acompañantes de los jóvenes, todas las estructuras sociales tienen la responsabilidad de consolidar procesos formativos y de control del uso de los videojuegos, además de ampliar los procesos de alfabetización digital.

\section{REFERENCIAS}

Asociación Española de Distribuidores y Editores de Software de Entretenimiento (ADESE) (2010). Informe anual año 2010. Barcelona.

Ayela, M. T. (2009). Adolescentes, transtornos de alimentación. San Vicente: Club Universitario.

Balerdi, F. (2011). Videojuegos violentos y agresividad. Pedagogia social, Revista Interuniversitaria (18), 31-39.

De la Cruz, Y. (2002). Videojuegos: generadores de actitudes ludópatas y violentas en los jugadores. Asamblea Legislativa de Costa Rica, San José.
Garrido, J. (2013). Videojuegos de estrategia: algunos principios para la enseñanza. Revista Electrónica de Investigación Educativa, 15(1), 62-74.

Gil, A., y Vida, M. (2007). Los videojuegos. Barcelona: ouc.

Gros, B. (2008). Videojuegos y aprendizaje. Barcelona: Editorial Grao.

Llorca, M. (2006). Los videojuegos, marcadores de tendencias en el ocio tecnológico. Revista Científica de Comunicación y Educación (27), 79-84.

Marqués, P. (2000). Las claves del éxito. Cuadernos de Pedagogía, 55-58.

Melo, M., y Hernández, R. (Septiembre-diciembre, 2014). El juego y sus posibilidades en la enseñanza de las ciencias naturales. Innovación Educativa, 14(66), 41-63.

Munné, F., y Cordina, N. (1996). Psicologia social del ocio y el tiempo libre. En J. L. Alvarado, A. Garrido y J. R. Torregrosa, Psicología social aplicada (pp. 429-448). Madrid: McGraw Hill.

Protégeles (2005). Videojuegos, menores y responsabilidad de los padres. Madrid: Protégeles.

Rojas, O. V. (Noviembre de 2008). Influencia de la televisión y videojuegos en el aprendizaje y conducta infanto-juvenil. Revista Chilena de Pediatría, 80-85.

Vigotsky, L. (2000). El desarrollo de los procesos psicológicos superiores. Barcelona: Crítica. 
